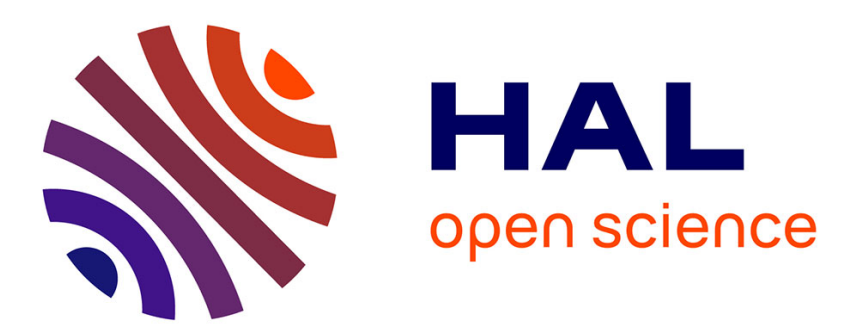

\title{
A Pen-Based Bimanual Approach for Interaction in Multi-Display Environments
}

Elio Keddisseh, Marcos Serrano, Emmanuel Dubois

\section{To cite this version:}

Elio Keddisseh, Marcos Serrano, Emmanuel Dubois. A Pen-Based Bimanual Approach for Interaction in Multi-Display Environments. 30eme conférence francophone sur l'interaction homme-machine, Oct 2018, Brest, France. 8p. hal-01899198

\section{HAL Id: hal-01899198 \\ https://hal.science/hal-01899198}

Submitted on 19 Oct 2018

HAL is a multi-disciplinary open access archive for the deposit and dissemination of scientific research documents, whether they are published or not. The documents may come from teaching and research institutions in France or abroad, or from public or private research centers.
L'archive ouverte pluridisciplinaire HAL, est destinée au dépôt et à la diffusion de documents scientifiques de niveau recherche, publiés ou non, émanant des établissements d'enseignement et de recherche français ou étrangers, des laboratoires publics ou privés. 


\section{Une approche bimanuelle basée sur un stylet pour l'interaction dans des environnements multi-écrans}

Elio Keddisseh

University of Toulouse - IRIT

31400, Toulouse, France

elio.keddisseh@irit.fr

\section{Marcos Serrano}

University of Toulouse - IRIT

31400, Toulouse, France

marcos.serrano@irit.f

Emmanuel Dubois

University of Toulouse - IRIT

31400, Toulouse, France

emmanuel.dubois@irit.fr

\begin{abstract}
Recent advances in multi-display environments (MDE) has led researchers to develop separated interaction techniques for separated MDE tasks. However, these techniques were not adapted to realistic usage situations. Additionally, when the use of a pen tablet is required, to annotate a document for example, its use in combination with the standard

keyboard and mouse often results in homing time cost. In this paper we explore a bimanual approach to support major MDE tasks: it is based on the combination of a multi-degree of freedom device and a pen. Moreover, we explain our approach and current implementation. Finally, we discuss our future work and envisioned studies to evaluate the usability of such an approach.
\end{abstract}

\section{Author Keywords}

Multi-display environments; Pen-based input; Bimanual input.

\section{CSS concepts}

Human-centered computing $\rightarrow$ Interaction design 


\section{Résumé}

Les avancées récentes dans les environnements multi-écrans (MDE) ont conduit les chercheurs à développer des

techniques d'interaction distinctes pour les différentes tâches effectuées dans ces environnements. Cependant, ces techniques sont souvent peu adaptéesà des situations d'utilisation réalistes. De plus, dans les situations où un stylet est requis pour prendre des notes par exemple, sa combinaison avec le clavier et la souris standard entraîne des surcoûts en termes de temps de changement de dispositifs. Dans cet article, nous explorons une approche bimanuelle permettant la prise en charge des principales tâches dans les MDE et basée sur le couplage d'un dispositif à multiples degrés de liberté et d'un stylet. Nous décrivons notre approche et sa mise en œuvre actuelle. Enfin, nous discutons nos futurs travaux et les études envisagées pour évaluer l'utilisabilité d'une telle approche.

\section{Mots Clés}

Environement multi-ecrans ; Interaction Bimanuelle

\section{Introduction}

Multi-display environments (MDEs) are now common and have advanced fairly quickly over the last few decades. One major factor contributing to this evolution is the variety of displays that exist and can be combined (e.g. electronic paper, tabletops, personal tablets, non-rectangular displays [1], etc.). Another factor that explains the use of MDEs is the advantages they bring, such as increasing user productivity and usability [2], and the ability of creating shared workspaces [3]. Moreover, MDEs can adopt many topologies [4] such as scattered displays in a room (meeting context), organized matrix (design, gaming) or horizontal setups (office). As a consequence, MDEs are used in many contexts, such as in meeting rooms [5], control rooms, shared workstations [6] and entertainment systems [7]. Moreover, in some of the aforementioned contexts, such as control and meeting rooms, users need to be able to take notes. Due to the benefits multimedia notes can have over a traditional paper [8] and the benefits of a pen over a keyboard [9], such users tend to use a pen tablet to take notes, thus complexifying even more the MDE environment.

With that diversity in MDEs setups and contexts, researchers have tackled the challenge of how to facilitate some frequent tasks in MDEs, such as monitor reaching [10] (the ability of selecting a display among the others), input redirection [11] (redirect input channels to different displays) and output redirection [12] (object transfer between displays). To better perform these tasks, researchers have either proposed new interaction techniques using existing input devices (pen [13][16]) or modified existing devices to adapt to one of these tasks (such as the Multi-Monitor Mouse [10] for display switching and Touch Projector [17] to interact with displays through a smartphone). However, most of the proposed techniques were not adapted to realistic usage situations [12] and are tackling only part of the MDE tasks [4]. For instance, in a control room context (Figure), where the user is sitting in front of a matrix of monitors, combining his pen tablet for annotations by his side, with keyboard and mouse to interact with a MDE results in a homing (device acquisition) time cost.

In this context, we explore the potential benefit of bimanua interaction to support common MDE tasks while preserving manual annotation with the dominant hand using a pen tablet. To this end, we propose the combination of TDome, a multi-DOF dome-like shaped mouse [4] specifically designed for MDEs, with a pen tablet. In this paper, we detail the concept, our implementation and the envisioned studies that will be carried to assess the usability of such an approach. 


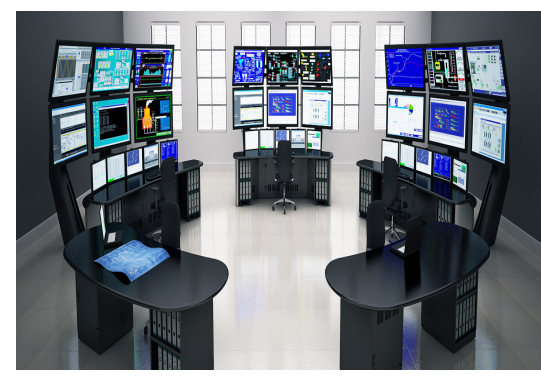

Figure 1: Control room setup "https://www.winsted.com/mark ets/oil-gas/"

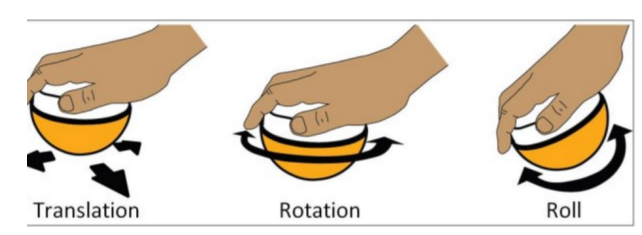

Figure 2: Physical manipulations that can be applied to TDome independently or in a combined way.

\section{Related work}

Some input devices were conceived for interacting with MDEs, yet none of these consider all major MDE tasks. For example, the multi-monitor mouse $\left(\mathrm{M}^{3}\right)$ simulates having one single cursor for every monitor in a multi-monitor setup, while using the mouse's side buttons to switch between screens. $\mathrm{M}^{3}$ was evaluated for display switching in a multimonitor setup [10] but does not tackle other MDE tasks.

Other interaction techniques were designed using existing input devices specifically for output redirection such as Pick'n Drop [14]: in this case it requires the user to touch the object with a digital pen to "pick it up" and touch again on the desired location to "drop it". However, this technique depends solely on the physical distribution of the displays in the MDE [12] and requires the user to move in the MDE to reach the desired display. Another technique is Radar View that displays a miniature environment of the surrounding MDE once the user touches the object with a digital pen. The user then drags the object to the desired location on the mini map. Once the pen is released, the object is transferred to the desired location in the MDE. Nonetheless, this technique lacks the ability of sensing the physical environment: it has to reconfigure the virtual environment every time the physical environment is updated.

Recently, Saidi et al. have designed a novel device, TDome, to address the major MDE tasks [4]. TDome is a half-sphere shaped mouse, topped with a touchscreen. Its bottomrounded shape provides 6 DOF (Rotation, Roll, Translation and Lift-Up (Figure ). The added touch screen on the top is used to (i) extend the device's gestures and to (ii) prevent any unintended physical manipulation. Moreover, TDome has the ability to sense the physical space making it independent from the MDE spatial setup. However, TDome was used and evaluated only with the dominant hand. Putting this in a control room context where users are already using the pen for annotations, they'll have to switch back and forth between TDome and the pen which will result in a homing effect as said earlier. Instead, in our work we explore how to use the 6 DOF of TDome in the non-dominant hand, in combination with a pen tablet in the dominant hand.

Additionally, researchers have used multi-DOF pens to design pen-tilt-based interaction techniques. For example, Tian et al. designed a menu selection interaction technique using a 'tilting' pen [19]. 'Tilt menu' resulted in less than $10 \%$ error rate when the pie menu was divided in 4 or 8 sections. Moreover, Xin et al. evaluated a pen-tilt-based selection technique [20] and found that subjects committed the fewest errors when the angular width was $30^{\circ}$. Similarly to TDome [4], a tilting pen allows efficient access to 6 commands. However, the multi-DOF pen was never tested in a MDE context.

\section{TDome + Pen: motivation and design factors}

Rational for the combination

In the context of smart and control rooms, the pen is frequently used for annotations. It thus has to be handled in the dominant hand. We know that people are able to perform contextual coarse positioning tasks with the non-dominant hand while the dominant hand performs detailed interactions [21]. Moreover, the non-dominant hand has at least the same performance than the dominant hand on coarse positioning tasks [22]. To complement the dominant hand interaction with the pen, the half-sphere shape of TDome and its 6 DOF could thus be used on the non-dominant hand to perform coarse positioning tasks. 


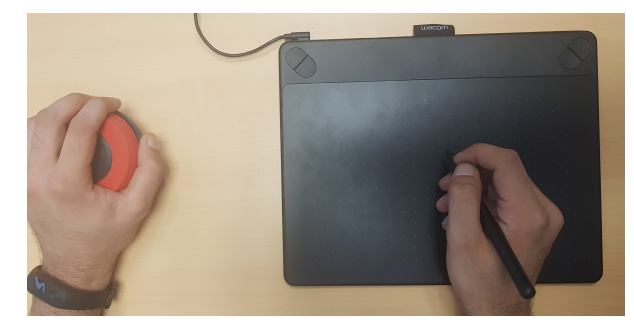

Figure 3: TDome + Pen
In our bimanual approach we therefore use TDome in the non-dominant hand to set context (e.g. reaching a display), and the pen in the dominant hand (Figure ) to perform a detailed interaction (e.g. positioning object in exact location), hence respecting Guiard's findings. In addition, the main functionalities of a keyboard - Typing and Accessing shortcuts - can be done with the pen and TDome respectively, thus, allowing us to exclude the keyboard from this technique and eliminating any homing time cost.

Using this bimanual approach, we designed several solutions to select a display in an MDE. This is a common and fundamental task, required prior to any other interaction in MDE environments. We adjusted the design of our interaction techniques in order to support display selection in three plausible perspectives, describing the setting of the user's interaction with regards to the selection in MDE environment: (1) egocentric, (2) device centric and (3) cursor centric. We also considered two different displays topologies. We hereafter describe these two considerations.

\section{Display selection techniques}

EGO CENTRIC

This perspective requires the device's axis that sets the context (TDome in our case) to be aligned with the user's axis. When performing an interaction in this point of view the cursor's initial position is the screen facing the user and it moves relatively to the user's position in the MDE. One implementation of this algorithm has been explored by Nacenta [23].

DEVICE CENTRIC

This perspective relies on the device's position in the MDE. We assume that it is a virtual device that can be fixed anywhere in the MDE. For example, the taskbar in Windows
OS is on the bottom of the screen by default and the user is free to place it either on one of the sides or the top. In our case, the cursor's initial position will be fixed in a defined location in the MDE.

\section{CURSOR CENTRIC}

This is the traditional approach of cursor detection, i.e. a cursor that can be displaced to the contiguous display. The starting point is always the display containing the cursor. This technique is efficient for selecting neighbor displays.

\section{MDE topologies}

A second consideration that drove our design is the MDE topology. We considered two different topologies that are relevant in the context of control or meeting rooms: (i) a matrix topology, where the user is sitting directly in front of 9 displays organized in a $3 \times 3$ matrix; (ii) an arc topology, where the user have displays next to him on each side in addition to a set of displays in front of him forming a semicircular form composed of 9 screens in total.

Display selection implementation

The general design approach of display selection technique in TDome + pen is a roll in the direction of the display with TDome followed by a pen tap on the tablet for confirmation which also represents the start of another task inside the selected display. For instance a roll in the north direction moves the display selecting cursor to the display in the north. If there is more than one display in that location, the amplitude of the roll determines what screen to be selected. To adapt the general technique to the perspective factors, we designed the following: 
Ego centric: The selecting cursor starts from the display facing the user's position and moves according to TDome's roll direction

(ii) Device centric: The selecting cursor starts from the pre-defined position of the device and moves according to TDome's roll direction

(iii) Cursor centric: The selecting cursor starts from the current display containing the cursor and moves according to TDome's roll direction for one display at a time. As opposed to other perspectives, the amplitude is not taken into consideration. The user has to clutch if a display exists between the starting point and the target display

Software and Hardware Implementation

In Figure we represent the 3 perspectives applied on the display selection technique. The blue rectangles represent the displays in the MDE. The highlighted borders in black represent the current cursor's location and the red rectangles are the target displays to be selected. In Ego centric, the cursor starts from screen 5 (the one facing the user's position). A slight roll to the east followed by a pen tap will select the next screen situated on the east (6). In Device centric, the cursor (device) is set at screen 1 . To select the target display (screen 9), the user should perform a hard roll in the south east direction. In Cursor centric, the cursor starts at screen 4 . To select the target display (screen 6 ) the user must go over screen 5 . Therefore, they should perform a slight roll to the east, reset TDome to its original position and then another slight roll to the east.

To detect the pen's data, we are using a Wacom Intuos 3D tablet that allows capturing in real time the physical $x$ and $y$ coordinates on tablet, virtual $x$ and $y$ coordinates of connected monitors, pressure and the buttons states of the pen (pressed and released). To detect TDome's physical manipulations, the device holds an X-IMU composed of a gyroscope, accelerometer and magnetometer that detects the rolls and rotations of the device.

We prototyped a GUI using C\# on visual studio that allows performing major MDE tasks with TDome. The GUI is made up from 9 panels representing the 9 proposed displays. Topology of the 9 display can be freely adjusted. Each pane contains one or more controls that can be selected, dragged inside the panel, and dragged and dropped in another panel.

For display selection (1), each panel can be highlighted when the user hover over it, followed by a validation gesture that change the panel's color. As for Output redirection (2) we implemented a drag and drop function that allows moving a control from one panel to another at the same location, followed by an adjustment to the exact target location. Input redirection (3) was prototyped by a cursor transfer to the new panel, whenever a new panel is selected.

With these implemented techniques, we are able to (1) use TDome + Pen to directly reach a display without the need of going through all existing displays between the starting point and the target; (2) to transfer objects from one display to another at an exact location using the two devices and without dragging it along the MDE; (3) to redirect the cursor to the selected screen immediately after selection and thus, removing time spent in MDE.

\section{Conclusion and future work}

In this work, we explore the use of TDome in the nondominant hand along with the pen in the dominant hand as a bimanual input technique to perform display selection in 
MDEs. Moreover, we have considered 3 frames of reference (Ego centric, Device centric and Cursor centric) for this technique and 2 display topologies.

A first perspective to this work will consist of designing and implementing the interaction techniques for MDE tasks, including display selection, input redirection and output redirection, and compare TDome + pen with keyboard + pen This comparison will allow us to (1) see if a multi-DOF mouse can be effective in the non-dominant hand, compared to keyboard shortcuts, and (2) evaluate bimanual interaction techniques for common tasks in MDEs. Moreover, after exploring the proposed design space, we can compare our technique with others from the literature on a real MDE setup and not on an MDE emulation.

Finally, concrete uses of this technique are expected to be developed in the context of control room and supervised situations such a as a teacher and multiple students running a simulation.

\section{Acknowledgment}

This work is funded by the AP2 project (research grant AP2 ANR-15-CE23-0001).

\section{References}

[1] M. Serrano, A. Roudaut, and P. Irani, "Investigating Text Legibility on Non-Rectangular Displays," in Proceedings of the 2016 CHI Conference on Human Factors in Computing Systems - CHI '16, 2016, pp. 498-508.

[2] J. Colvin, N. Tobler, and J. A. Anderson, "31-53 Productivity and Multi-Screen Displays 54-60 An Essential Guide for Sound Health Communication Campaign Development: A Review of Effective Health
Risk Messages: A Step-by-Step Guide Lindsey Polonec," 2004.

[3] J. R. Wallace, S. D. Scott, T. Stutz, T. Enns, and K Inkpen, "Investigating teamwork and taskwork in single- and multi-display groupware systems," Pers. Ubiquitous Comput., vol. 13, no. 8, pp. 569-581, Nov. 2009.

[4] H. Saidi, M. Serrano, P. Irani, and E. Dubois, "TDome," in Proceedings of the 2017 CHI Conference on Human Factors in Computing Systems - CHI '17, 2017, pp. 5892-5904.

[5] P. Chiu et al., "Manipulating and annotating slides in a multi-display environment," PROC. INTERACT '03, pp. 583--590, 2003.

[6] C. Shen, K. Everitt, and K. Ryall, "UbiTable: Impromptu Face-to-Face Collaboration on Horizontal Interactive Surfaces," Springer, Berlin, Heidelberg, 2003, pp. 281-288.

[7] M. A. Nacenta and M. A. Nacenta, "1 Inventing the Future of Multi-Display Environments (position paper)."

[8] P. Chiu, A. Kapuskar, S. Reitmeier, and L. Wilcox, "NoteLook," in Proceedings of the seventh ACM international conference on Multimedia (Part 1) MULTIMEDIA '99, 1999, pp. 149-158.

[9] P. A. Mueller and D. M. Oppenheimer, "The Pen Is Mightier Than the Keyboard," Psychol. Sci., vol. 25, no. 6, pp. 1159-1168, Jun. 2014. 
[10] H. Benko and S. Feiner, "Multi-monitor mouse," in $\mathrm{CHI}$ '05 extended abstracts on Human factors in computing systems - CHI '05, 2005, p. 1208.

[11] B. Johanson, G. Hutchins, T. Winograd, and M. Stone, "PointRight," in Proceedings of the 15th annual ACM symposium on User interface software and technology - UIST '02, 2002, p. 227.

[12] M. A. Nacenta, D. Aliakseyeu, S. Subramanian, and C. Gutwin, "A comparison of techniques for multi-display reaching," in Proceedings of the SIGCHI conference on Human factors in computing systems - $\mathrm{CHI}$ '05, 2005, p. 371.

[13] J. Rekimoto, Y. Ayatsuka, and M. Kohno, "SyncTap: An Interaction Technique for Mobile Networking," Springer, Berlin, Heidelberg, 2003, pp. 104-115.

[14] J. Rekimoto and Jun, "Pick-and-drop," in Proceedings of the 10th annual ACM symposium on User interface software and technology - UIST '97, 1997, pp. 3139.

[15] P. Baudisch et al., "Drag-and-Pop and Drag-and-Pick: Techniques for Accessing Remote Screen Content on Touch- and Pen-Operated Systems," HumanComputer Interact. '03, Jan. 2003.

[16] D. Aliakseyeu, M. A. Nacenta, S. Subramanian, and C. Gutwin, "Bubble radar," in Proceedings of the working conference on Advanced visual interfaces - AVI '06, 2006, p. 19.

[17] S. Boring, D. Baur, A. Butz, S. Gustafson, and P.
Baudisch, "Touch projector," in Proceedings of the 28th international conference on Human factors in computing systems - CHI'10, 2010, p. 2287.

[18] M. Waldner, E. Kruijff, and D. Schmalstieg, "Bridging gaps with pointer warping in multi-display

environments," in Proceedings of the 6th Nordic Conference on Human-Computer Interaction Extending Boundaries - NordiCHI'10, 2010, p. 813.

[19] F. Tian et al., "Tilt menu," in Proceeding of the twenty-sixth annual CHI conference on Human factors in computing systems - CHI '08, 2008, p. 1371.

[20] Y. Xin, X. Bi, and X. Ren, "Acquiring and pointing," in Proceedings of the 2011 annual conference on Human factors in computing systems - CHI '11, 2011, p. 849.

[21] Y. Guiard, "Asymmetric division of labor in human skilled bimanual action: the kinematic chain as a model.," J. Mot. Behav., vol. 19, no. 4, pp. 486-517, Dec. 1987.

[22] P. Kabbash, I. S. MacKenzie, and W. Buxton, "Human performance using computer input devices in the preferred and non-preferred hands," in Proceedings of the SIGCHI conference on Human factors in computing systems - CHI'93, 1993, pp. 474-481.

[23] M. A. Nacenta, S. Sallam, B. Champoux, S. Subramanian, and C. Gutwin, "Perspective cursor," in Proceedings of the SIGCHI conference on Human Factors in computing systems - CHI'06, 2006, p. 289. 
